\title{
A successful pregnancy using completely immotile but viable frozen-thawed spermatozoa selected by
} laser

\author{
Huanhua Chen ${ }^{1 *}$, Guixue Feng ${ }^{1,2 *}$, Bo Zhang ${ }^{1}$, Hong Zhou', Jinhui Shu' ${ }^{1}$ Xianyou Gan ${ }^{1}$ \\ ${ }^{1}$ Center for Reproductive Medicine, The Maternal and Child Health Hospital of Guangxi Zhuang Autonomous Region, Nanning; ${ }^{2}$ Center for \\ Reproductive Medicine, The Obstetrics and Gynecology Hospital of Guangxi Zhuang Autonomous Region, Nanning, China
}

The aim of this study was to report a successful pregnancy using completely immotile frozen-thawed spermatozoa selected by laser. A single laser shot was used to detect the presence of viable immotile spermatozoa in fresh and frozen-thawed testicular spermatozoa. The viability rate was 55.8\% after the laser detection, and cryopreservation was carried out immediately. The thawing test was performed on the day of oocyte pick-up, and no motile sperm were observed after extending the culture for another 4 hours, while a survival rate of $39.8 \%$ was detected using the laser. In all, five mature oocytes were injected, resulting in four cases of normal fertilization (80\%) on day 1. Further, two high-quality day 3 embryos were transferred, which resulted in a singleton pregnancy. Our study demonstrates that completely immotile spermatozoa are worth cryopreserving for further intracytoplasmic sperm injection, which provides a new insight into male fertility preservation in cases of completely immotile spermatozoa.

Keywords: Cryopreservation; Immotile spermatozoa; Intracytoplasmic sperm injections; Lasers

\section{Introduction}

Intracytoplasmic sperm injection (ICSI) has emerged as an ideal tool for the treatment of severe male infertility. Previous studies have confirmed that a viable spermatozoon is the prerequisite for initiating fertilization in cases of ICSI [1]. Motility is the primary sign used for determining the viability of a spermatozoon. It was reported that ICSI using completely immotile spermatozoa usually resulted in a rel-

Received: Oct 20, 2016 · Revised: Jan 19, 2017 · Accepted: Jan 19, 2017 Corresponding author: Bo Zhang

Reproductive Medicine Center, Maternal and Child Health Hospital of Guangxi Zhuang Autonomous Region, No. 225, Xinyang Road, Nanning 530003, China Tel: +86-771-5802003 Fax:+86-771-5802003 E-mail: cestbon269@sina.com

*These authors contributed equally to this study.

*This study was supported by the research fund of National Health and Family Planning Commission of China (201402004), Guangxi National Science Foundation (2013 GXNSFAA019258), and Key Scientific Research Project of Medical and Health in Guangxi (Zhong2011063, Zhong 2011065, Z2012210).

This is an Open Access article distributed under the terms of the Creative Commons Attribution Non-Commercial License (http://creativecommons.org/licenses/by-nc/4.0/) which permits unrestricted non-commercial use, distribution, and reproduction in any medium, provided the original work is properly cited. atively low fertilization rate [2] and at times, even complete fertilization failure [3]. Therefore, motile sperm are preferentially selected for ICSI in practice. Thus far, sperm cryopreservation is the most effective method of preserving male fertility, although the freezing-thawing process can cause irreversible damage to the sperm [4]. Considering the cryodamage to the sperm, only motile sperm should be cryopreserved. However, we do not know whether completely immotile spermatozoa are suitable for cryopreservation for ICSI. On the basis of our previous reports, we present a case in which successful pregnancy was achieved by using a noncontact diode laser (wavelength, $1.48 \mu \mathrm{m}$ ) to identify viable sperm before freezing and after thawing in a patient with completely immotile spermatozoa; this may help embryologists recognize the value of cryopreserving completely immotile spermatozoa.

\section{Case report}

\section{Patient history}

In this study, we considered a 40-year-old man with infertility due to 
nonobstructive azoospermia. His 38-year-old wife presented to Center for Reproductive Medicine, The Maternal and Child Health Hospital of Guangxi Zhuang Autonomous Region with a 10-year-long history primary infertility. The man could anterogradely ejaculate without impotence or premature ejaculation. His serum follicle-stimulating hormone level was $14.7 \mathrm{mIU} / \mathrm{mL}$, and his karyotype was 46,XY without $Y$-chromosome microdeletion. A semen analysis according to the World Health Organization manual [5] repeatedly showed azoospermia. Three to five completely immotile spermatozoa per view were observed in the retrieval of the extracted testicular sperm under an inverted microscope (magnification $\times 200$; Olympus, Tokyo, Japan) after culture for 4 hours. Sperm viability was then assessed by applying a laser beam directly to the tip of the sperm flagellum. The viable spermatozoa rate was $55.8 \%$, which suggested that this spermatozoa sample was worth freezing for subsequent ICSI.

\section{Testicular sperm aspiration and cryopreservation}

The patient was placed in the supine position and disinfected following routine procedures. Anesthesia was performed by $2 \%$ lidocaine to the spermatic cord block. A 50-mL syringe containing $0.5 \mathrm{~mL}$ of Quinn's 1020 (Sage, Trumbull, CT, USA) and a 16-gauge needle were used for each sample. These samples were then independently minced using two sterile needles in a dish containing $2 \mathrm{~mL}$ of the fertilization medium, Quinn's 1020. The sperm sample containing a 200 $\mu \mathrm{L}$ suspension was concentrated to $5 \mu \mathrm{L}$ by centrifugation $(2,000$ rpm) and then diluted with a sperm cryoprotectant medium in a 1:1 ratio. After being maintained at the liquid nitrogen surface for $4 \mathrm{~min}$ utes, the cryotip was rapidly immersed in liquid nitrogen at $-196^{\circ} \mathrm{C}$ for long-term cryopreservation.

The thawing test was performed on the day of oocyte pick-up as follows: the cryotip was taken out of the liquid nitrogen, placed immediately into the $37^{\circ} \mathrm{C}$ water bath, and kept for 15 minutes before adding to the Quinn's 1020 dish. After incubation for 4 hours, no motile sperm were observed, and viability was assessed by laser.

\section{Ovarian stimulation protocols and oocyte collection}

Controlled ovarian stimulation was carried out after downregulation with Diphereline (Ipsen, Tianjin, China). Follicle-stimulating hormone was administered at a dose of $200 \mathrm{IU}$ per day as described previously by Feng et al. [6]. When dominant follicles reached a diameter of $18 \mathrm{~mm}$, ovulation was induced by an intramuscular injection of 10,000 IU of human chorionic gonadotrophin (hCG). Oocytes were recovered by the transvaginal aspiration of follicles under ultrasound guidance 36 hours after the hCG injection.

\section{Laser-assisted selection of immotile spermatozoa}

Sperm viability was assessed by a diode laser (wavelength, 1.48

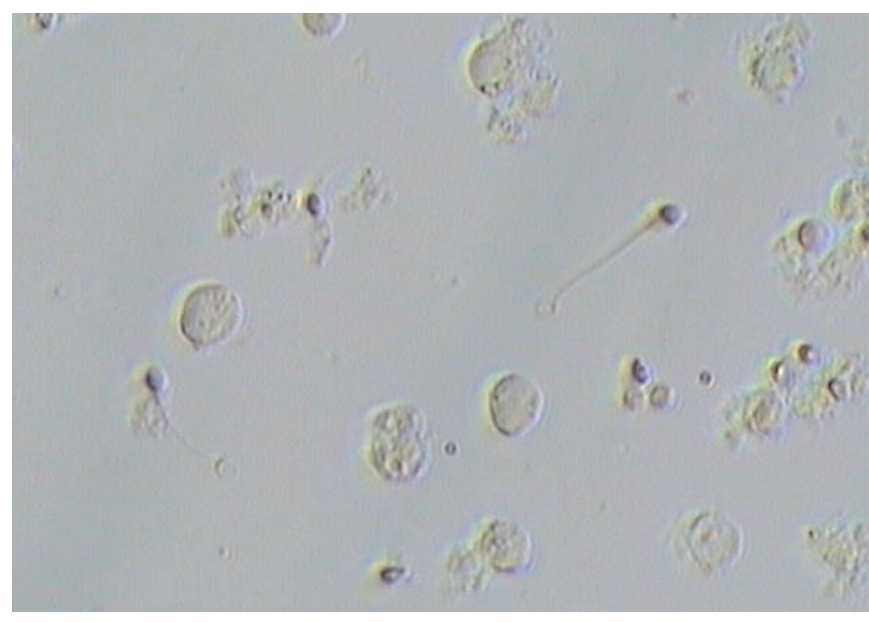

Figure 1. A single laser shot was applied towards the end of the tail of immotile testicular spermatozoa; the tail of the viable spermatozoa started curling within a few seconds after the laser shot.

$\mu \mathrm{m})$ as described by Aktan et al. [7]. Before the freezing process, testicular sperm viability was assessed by laser, and cryopreservation was performed immediately. Thawed spermatozoa were washed, and the pellet was suspended in Quinn's 1020 medium. Laser assessment of sperm viability was performed by a direct laser shot to the tip of the sperm flagellum using laser energy of approximately 200 $\mu \mathrm{J}$ at an irradiation time of approximately $2 \mathrm{~ms}$. If a spermatozoon responded to the laser shot by the curling of its tail, it was regarded as presumably viable and selected for ICSI (Figure 1).

\section{Embryo culture and pregnancy determination}

Fertilization was checked by the detection of two pronuclei and two polar bodies at 16 to 18 hours after ICSI. Only zygotes displaying two pronuclei were transferred to Quinn's 1026 medium (Sage) supplemented with $10 \%$ serum protein substitute (Sage) from days 0 to 3, and then were moved into Quinn's 1029 medium (Sage) supplemented with $10 \%$ serum protein substitute for another 2 days. A biochemical pregnancy test was carried out 14 days after embryo transfer by measuring the serum $\beta$-hCG level. Clinical pregnancy was confirmed if a gestational sac was observed in a vaginal ultrasound 6 to 8 weeks after transfer.

On the day of oocyte pick-up, the spermatozoa sample was thawed and no motile spermatozoa were detected, while $39.8 \%$ of the spermatozoa were observed to be viable in the laser assessment. After culturing for 4 hours, the viable spermatozoa from the frozen-thawed immotile spermatozoa were selected by laser for ICSI. A total of five mature oocytes were injected, resulting in four cases of normal fertilization ( $80 \%$ ) on day 1 , four cases of normal cleavage on day 2 , and two high-quality embryos on day 3. Two high-quality embryos were chosen for transfer under ultrasound guidance. Fourteen days after 
the intrauterine transfer, the blood concentration of $\beta$-hCG was 492.6 $\mathrm{IU} / \mathrm{L}$, and one gestational sac with heart activity was observed by transvaginal ultrasonography 3 weeks after the transfer.

\section{Discussion}

To the best of our knowledge, this is the first report of a successful pregnancy achieved using completely immotile frozen-thawed spermatozoa selected by laser. Our report demonstrates for the first time that completely immotile sperm can be successfully frozen and that normal in vitro fertilization (IVF) ability can be achieved, which may provide a new insight into male fertility preservation in patients with completely immotile sperm.

Regarding the cryodamage to the sperm, only motile sperm are generally cryopreserved in routine clinical operations [8], while the immotile spermatozoa are often discarded, resulting in the loss of the opportunity of assisted reproductive treatment for some patients. Therefore, effective preservation of the fertility of viable but immotile spermatozoa is a major challenge facing IVF. As is known, completely immotile spermatozoa are often found in IVF treatment cycles involving ejaculate samples in patients with conditions such as primary cilia dyskinesia [9-11], which is a rare autosomal recessive multisystem disorder characterized by ultrastructural and functional defects of the ciliated cells of the body. Completely immotile spermatozoa also occasionally occur in surgically extracted testicular spermatozoa [12], which can be attributed to very heterogeneous events and are a consequence of oxidative stress, infection, or numerous other factors [13]. According to the traditional freezing procedure, immotile testicular spermatozoa are likely to be abandoned and a subsequent biopsy is scheduled, which increases both the chances of long-term complications and the economic burden on the patients. Many studies have demonstrated that using fresh immotile sperm for ICSI can lead to successful fertilization $[9,12]$. However, it is unclear whether immotile spermatozoa are worth cryopreserving for further ICSI. The selection of immotile but viable spermatozoa on the day of ICSI is a major challenge for embryologists [14].

Several complementary approaches have been developed for detecting immotile but viable sperm. The latest World Health Organization manual (2010) for the examination and processing of human semen recommends the eosin test [5], which may be used in combination with negrosin, and the hypo-osmotic swelling (HOS) test for the diagnostic evaluation of vitality. The eosin test is the standard technique for determining sperm viability and is based on the exclusion of the dye. Nonviable spermatozoa exhibit deficient cell membrane structures, permitting eosin to enter the cells and stain them, whereas viable spermatozoa remain unstained. However, once exposed to toxic dyes, spermatozoa are no longer suitable for ICSI, which limits its application in assisted reproductive techniques [15]. The HOS test has been reported to be successful in the fertility treatment of patients with fresh completely immotile spermatozoa [16]. However, it must be noted that spontaneously developed tail swelling may influence the accuracy of the HOS test in the determination of the membrane integrity and viability of human spermatozoa [17]. Therefore, this method is not suitable for spermatozoa that have been processed, specifically for cryopreservation, and then thawed. A healthy birth was reported after the laser-assisted immobilization of spermatozoa before ICSI [18]. Application of a noncontact diode laser to immobilize sperm is considered safe, because its energy is much lower than that used in assisted hatching [19]. In 2004, Aktan et al. [7] for the first time presented data showing that presumably viable spermatozoa can be easily selected by using a laser system. Their results showed that the percentage of immotile spermatozoa classified as viable by the laser test $(22.0 \%)$ was similar to that detected by the HOS test $(21.50 \%)$. The fertilization rate increased significantly from $20.4 \%$ in the randomly selected testicular sperm extraction spermatozoa to $45.4 \%$ in the laser-selected group; accordingly, the takehome baby rate increased from $5.9 \%$ to $19.0 \%$. Laser-assisted selection of viable spermatozoa in a patient with completely immotile sperm, which were subsequently used for ICSI, was found to lead to a successful pregnancy $[9,20]$. Compared with the HOS test, laser-assisted selection of viable sperm is more objective, simple, and rapid, and does not require the use of other chemical agents. Here, we described the successful cryopreservation of immotile but viable spermatozoa detected by single laser shots, and the achievement of ideal embryo development and clinical outcomes using the viable frozenthawed spermatozoa for ICSI.

In conclusion, we believe that viable but immotile spermatozoa can be preserved for further ICSI, which provides a new insight into male fertility preservation with completely immotile spermatozoa.

\section{Conflict of interest}

No potential conflict of interest relevant to this article was reported.

\section{References}

1. Hessel M, Robben JC, D'Hauwers KW, Braat DD, Ramos L. The influence of sperm motility and cryopreservation on the treatment outcome after intracytoplasmic sperm injection following testicular sperm extraction. Acta Obstet Gynecol Scand 2015;94: 1313-21.

2. Stalf T, Mehnert C, Hajimohammad A, Manolopoulos K, Shen Y, Schuppe HC, et al. Influence of motility and vitality in intracytoplasmic sperm injection with ejaculated and testicular sperm. 
Andrologia 2005;37:125-30.

3. Javed M, Esfandiari N, Casper RF. Failed fertilization after clinical intracytoplasmic sperm injection. Reprod Biomed Online 2010; 20:56-67.

4. John Morris G, Acton E, Murray BJ, Fonseca F. Freezing injury: the special case of the sperm cell. Cryobiology 2012;64:71-80.

5. World Health Organization. WHO laboratory manual for the examination and processing of human semen. 5th ed. Geneva: World Health Organization; 2010.

6. Feng G, Zhang B, Zhou H, Shu J, Gan X, Wu F, et al. Comparable clinical outcomes and live births after single vitrified-warmed and fresh blastocyst transfer. Reprod Biomed Online 2012;25: 466-73.

7. Aktan TM, Montag M, Duman S, Gorkemli H, Rink K, Yurdakul T. Use of a laser to detect viable but immotile spermatozoa. Andrologia 2004;36:366-9.

8. Desch L, Bruno C, Herbemont C, Michel F, Bechoua S, Girod S, et al. Impact on ICSI outcomes of adding $24 \mathrm{~h}$ of in vitro culture before testicular sperm freezing: a retrospective study. Basic Clin Androl 2015;25:6.

9. Gerber PA, Kruse R, Hirchenhain J, Krussel JS, Neumann NJ. Pregnancy after laser-assisted selection of viable spermatozoa before intracytoplasmatic sperm injection in a couple with male primary cilia dyskinesia. Fertil Steril 2008;89:1826.e9-12.

10. Geber S, Lemgruber M, Taitson PF, Valle M, Sampaio M. Birth of healthy twins after intracytoplasmic sperm injection using ejaculated immotile spermatozoa from a patient with Kartagener's syndrome. Andrologia 2012;44 Suppl 1:842-4.

11. Montjean D, Courageot J, Altie A, Amar-Hoffet A, Rossin B, Geoffroy-Siraudin $C$, et al. Normal live birth after vitrified/warmed oocytes intracytoplasmic sperm injection with immotile spermatozoa in a patient with Kartagener's syndrome. Andrologia 2015;47:839-45.
12. Nordhoff V, Schuring AN, Krallmann C, Zitzmann M, Schlatt S, Kiesel L, et al. Optimizing TESE-ICSI by laser-assisted selection of immotile spermatozoa and polarization microscopy for selection of oocytes. Andrology 2013;1:67-74.

13. Ortega C, Verheyen G, Raick D, Camus M, Devroey P, Tournaye H. Absolute asthenozoospermia and ICSI: what are the options? Hum Reprod Update 2011;17:684-92.

14. Nordhoff V. How to select immotile but viable spermatozoa on the day of intracytoplasmic sperm injection? An embryologist's view. Andrology 2015;3:156-62.

15. Buckett WM. Predictive value of hypo-osmotic swelling test to identify viable non-motile sperm. Asian J Androl 2003;5:209-12.

16. Tubman A, Check JH, Bollendorf A, Wilson C. Effect of poor motility on pregnancy outcome following intracytoplasmic sperm injection in couples whose male partners have subnormal hypoosmotic swelling test scores. Clin Exp Obstet Gynecol 2013;40: 315-6.

17. Hossain A, Osuamkpe C, Hossain S, Phelps JY. Spontaneously developed tail swellings (SDTS) influence the accuracy of the hypo-osmotic swelling test (HOS-test) in determining membrane integrity and viability of human spermatozoa. J Assist Reprod Genet 2010;27:83-6.

18. Ebner T, Moser M, Yaman C, Sommergruber M, Tews G. Successful birth after laser assisted immobilization of spermatozoa before intracytoplasmic injection. Fertil Steril 2002;78:417-8.

19. Ebner T, Yaman C, Moser M, Sommergruber M, Hartl J, Tews G. Laser assisted immobilization of spermatozoa prior to intracytoplasmic sperm injection in humans. Hum Reprod 2001;16:262831.

20. Chen HH, Feng GX, Zhang B, Shu JH, Gan XY, Zhou H, et al. Successful pregnancy following laser-assisted selection of viable but immotile spermatozoa for intracytoplasmic sperm injection: a report of 2 cases. Zhonghua Nan Ke Xue 2015;21:988-91. 